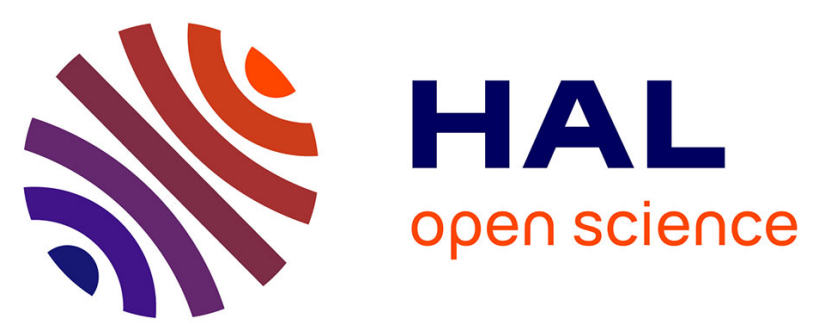

\title{
Using thickness-shear mode quartz resonator for characterizing the viscoelastic properties of PDMS during cross-linking, from the liquid to the solid state and at different temperatures
}

\author{
Amaury Dalla Monta, Florence Razan, Jean-Benoit Le Cam, Grégory
}

Chagnon

\section{To cite this version:}

Amaury Dalla Monta, Florence Razan, Jean-Benoit Le Cam, Grégory Chagnon. Using thickness-shear mode quartz resonator for characterizing the viscoelastic properties of PDMS during cross-linking, from the liquid to the solid state and at different temperatures. Sensors and Actuators A: Physical, 2018, 280, pp.107-113. 10.1016/j.sna.2018.07.003 . hal-01874677

\section{HAL Id: hal-01874677}

\section{https://hal-univ-rennes1.archives-ouvertes.fr/hal-01874677}

Submitted on 30 Jan 2019

HAL is a multi-disciplinary open access archive for the deposit and dissemination of scientific research documents, whether they are published or not. The documents may come from teaching and research institutions in France or abroad, or from public or private research centers.
L'archive ouverte pluridisciplinaire HAL, est destinée au dépôt et à la diffusion de documents scientifiques de niveau recherche, publiés ou non, émanant des établissements d'enseignement et de recherche français ou étrangers, des laboratoires publics ou privés. 


\title{
Using thickness-shear mode quartz resonator for characterizing the viscoelastic properties of PDMS during cross-linking, from the liquid to the solid state and at different temperatures
}

\author{
Amaury Dalla Monta ${ }^{\mathrm{a}, \mathrm{b}}$, Florence Razan ${ }^{\mathrm{a}}$, \\ Jean-Benoît Le Cam ${ }^{\mathrm{b}, \mathrm{c}, 1}$, Grégory Chagnon ${ }^{\mathrm{d}, \mathrm{e}}$

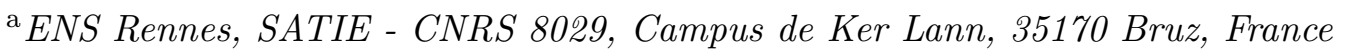 \\ ${ }^{\mathrm{b}}$ Univ Rennes, CNRS, IPR (Institut de Physique de Rennes) - UMR 6251, \\ F-35000 Rennes, France \\ ${ }^{\mathrm{c}} L C$-DRIME, Joint Research Laboratory in Imaging, Mechanics and Elastomers, \\ University of Rennes 1/Cooper Standard/CNRS, Campus de Beaulieu, Bât. 10B, \\ F-35042 Rennes Cedex, France \\ ${ }^{\mathrm{d}}$ Université Grenoble Alpes, TIMC-IMAG, F-38000 Grenoble, France \\ ${ }^{\mathrm{e}}$ CNRS, TIMC-IMAG, F-38000 Grenoble, France
}

\begin{abstract}
Characterizing the effects of cross-linking level and kinetics on the mechanical properties of rubber, especially viscoelasticity, provides information of importance to better understand and predict final mechanical properties. Classically, the effects of cross-linking on the mechanical properties are investigated with a rheometer. Typical results give the evolution of elastic properties of rubber in the solid state with respect to time or cross-linking level. In the case where the rubber is initially in the liquid state, such as some silicone rubbers, this type of characterization is not suitable anymore. In this study, the Quartz Crystal Microbalance (QCM) principle has been used in order to characterize the viscoelastic properties (elastic and viscous moduli) of a silicone rubber during cross-linking, from the liquid (uncross-linked) to the solid (final cross-linked) state. The device consists in a Thickness-Shear Mode (TSM) resonator generating ultrasonic waves, which provides viscoelastic properties of a material in contact with its surface from an electrical impedance analysis. In contrast to other characterization tools, it makes possible the continuous and nondestructive characterization of viscoelastic properties from a small material volume, under $1 \mathrm{~mL}$. It has been used to characterize the evolution of the viscoelastic proper-
\end{abstract}


ties of a polydimethylsiloxane during cross-linking at three different temperatures: $25^{\circ} \mathrm{C}, 50{ }^{\circ} \mathrm{C}$ and $80{ }^{\circ} \mathrm{C}$. Results have shown that the final viscoelastic properties depend on the temperature during crosslinking. The higher the crosslinking temperature, the lower (higher) the final elastic (viscous) modulus at $25{ }^{\circ} \mathrm{C}$. This is 
explained by the strong difference in the cross-linking kinetics obtained with the temperatures applied.

Key words: Quartz Crystal Microbalance, liquid and solid states, cross-linking, viscoelasticity, PDMS

\section{Introduction}

The Quartz Crystal Microbalance (QCM) is a versatile tool, first described by Sauerbrey in his seminal work (Sauerbrey, 1959). Due to its simplicity, this type of sensor has been widely used in various domains, enabling us to determine with accuracy measurands (Cheeke and Wang, 1999) such as mass density (Stockbridge, 1966b), viscosity (Kanazawa and Gordon, 1985) and pressure (Stockbridge, 1966a), in a continuous and non-destructive manner, with sample as small as a microliter. As illustrated in Figure 1, it consists in a circular thin disk of piezoelectric ATcut quartz, with metallic electrodes on both sides. The application of a voltage between them generates a shear deformation of the crystal, which can then be excited into resonance when its thickness is near an odd multiple of half the acoustic wavelength. The deposit of a material sample on the crystal changes the resonance properties, which are fundamentally dependent on the characteristics, either mechanical or electrical, of the sample. For all its applications, the QCM needs an electrical interface able to apply a sinusoidal voltage between its electrodes and to measure the resonance conditions. Among all the methods of read-out, the most used is the impedance analysis (Arnau, 2008), which maps the electrical admittance of the QCM as a function of the frequency, giving access to a greater number of parameters than simpler methods such as the use of an oscillator circuitry.

In biology, where the QCM is increasingly used (Becker and Cooper, 2011), the functionalization of the QCM surface with a definite substance enables us to measure active species absorption or deposition, and then to recognize specific pathologies like schistosomiasis (Wang et al., 2006) or Ebola fever (Yu et al., 2006). In chemistry, with a similar method, such a sensor can detect presence of harmful molecules in the air, acting as an electronic nose (Si et al., 2007). In mechanics, finally, it is used for measuring complex shear modulus of polymers (Lucklum et al., 2000; Holt et al., 2006; Serfaty et al., 2012) or for following its evolution during a

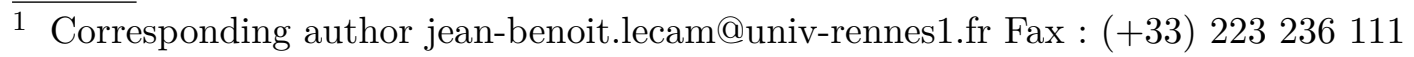


specific process, such as dissolution (Hinsberg et al., 1986).

To our best knowledge, the QCM has never been used to characterize the viscoelastic properties of a rubber at different stages of the crosslinking, while it should provide additional information to better describe and understand crosslinking kinetics. This study aims at performing such measurements with a polydimethylsiloxane, from the liquid to the solid state, at different temperatures.

The paper is organized as follows. Section 2 briefly describes the theory governing the behavior of the QCM and the measurement principle. Section 3 presents the application to the characterization of viscoelasticity of a polydimethylsiloxane: the experimental device, the measurement methodology and the material. Results obtained are analyzed in light of the aforementioned objectives. Concluding remarks close the paper.

\section{Theoretical framework and measurement principle}

On a fundamental point of view, the QCM is simply a transducer, linking its load impedance with its electrical impedance. The fundamental relations governing its behavior are briefly recalled hereafter. The reader can refer to Johannsmann (2015) for further information.

\subsection{Acoustic parameterization}

In a first approximation, when its diameter is large compared to its thickness, the QCM can mainly be seen as a unidimensional device, a succession of homogeneous layers in which acoustic shear waves propagate along the $\mathrm{z}$ axis. Inside a layer, the amplitude $u(z, t)$ of the displacement due to the wave, $z$ and $t$ being respectively the $\mathrm{z}$ coordinate and the time, is described by the well-known wave equation, which is:

$$
\frac{\partial^{2} u}{\partial t^{2}}=c^{2} \frac{\partial^{2} u}{\partial z^{2}}
$$


This can be expressed in the frequency domain by:

$$
-\omega^{2} \hat{u}(z)=\tilde{c}^{2} \frac{\mathrm{d}^{2} \hat{u}}{\mathrm{~d} z^{2}}
$$

Here, $\hat{u}$ is the complex amplitude of the displacement, $\tilde{c}=\sqrt{\rho / \tilde{G}}$ is the speed of sound, $\rho$ the density and $\tilde{G}$ the shear modulus. Introducing the wavenumber $\tilde{k}=\sqrt{\omega / \tilde{c}}$ and the imaginary unit $i$, the solutions of the wave equation are in the form of:

$$
\hat{u}(z)=\hat{u}^{+} \exp (+i \hat{k} z)+\hat{u}^{-} \exp (-i \hat{k} z)
$$

$\hat{u}^{+}$and $\hat{u}^{-}$are the amplitude of a wave traveling respectively to the left and to the right. It is therefore possible to express the velocity $\hat{v}(z)$ and the shear stress $\hat{\sigma}(z)$ :

$$
\begin{aligned}
\hat{v}(z) & =i \omega \hat{u}(z) \\
& =i \omega \hat{u}^{+} \exp (+i \hat{k} z)+i \omega \hat{u}^{-} \exp (-i \hat{k} z) \\
\hat{\sigma}(z) & =\tilde{G} \frac{\mathrm{d} \hat{u}}{\mathrm{~d} z} \\
& =i \tilde{k} \tilde{G} \hat{u}^{+} \exp (+i \hat{k} z)-i \tilde{k} \tilde{G} \hat{u}^{-} \exp (-i \hat{k} z) \\
& =i \omega \tilde{Z} \hat{u}^{+} \exp (+i \hat{k} z)-i \omega \tilde{Z} \hat{u}^{-} \exp (-i \hat{k} z)
\end{aligned}
$$

It is important to notice that the acoustic wave impedance $\tilde{Z}=\rho \tilde{c}$ is a mechanical property of the layer, and should not be confused with the acoustic impedance, the ratio between shear stress and velocity $\tilde{Z}_{a c}=\hat{\sigma}(z) / \hat{v}(z)$, which is characteristic of the excitation.

\subsection{Mason circuit}

The acoustic shear wave is in nature very similar to an electromagnetic wave. By virtue of the electromechanical analogy, it is therefore possible to represent a layer of the QCM as a distributed-element network. Such a representation is perfectly adapted to this device, because our goal is to link a mechanical quantity with 
an electrical quantity. To go further, since only the quantities at the interfaces between two layers are of interest, the QCM can even be represented as a two-port network, one for each interface (see Figure 2(a)). This paragraph shows that the Mason circuit is a compatible representation (Mason and Baerwald, 1951).

Here, $A$ is the area of the device in the plane orthogonal to the direction of propagation, and $h$ is half the thickness of the considered layer. Using the Kirchhoff rules, it appears that forces and velocities are related by the following expressions that should be validated:

$$
\begin{aligned}
& \hat{F}_{1}=i A \tilde{Z} \tan (\tilde{k} h) \hat{v}_{1}-\frac{i A \tilde{Z}}{\sin (2 \tilde{k} h)}\left(\hat{v}_{1}+\hat{v}_{2}\right) \\
& \hat{F}_{2}=\frac{i A \tilde{Z}}{\sin (2 \tilde{k} h)} \hat{v}_{1}-i A \tilde{Z} \tan (\tilde{k} h)\left(\hat{v}_{1}+\hat{v}_{2}\right)
\end{aligned}
$$

The displacement and the shear stress can be written as:

$$
\begin{aligned}
& \hat{u}(z)=\hat{u}_{\alpha} \sin (\hat{k} z)+\hat{u}_{\beta} \cos (\hat{k} z) \\
& \hat{\sigma}(z)=\omega \tilde{Z}\left(\hat{u}_{\alpha} \sin (\hat{k} z)+\hat{u}_{\beta} \cos (\hat{k} z)\right)
\end{aligned}
$$

Hence, being careful about the sign of the relations:

$$
\begin{array}{ll}
\hat{F}_{1}=-A \hat{\sigma}(-h) & \hat{v}_{1}=+i \omega \hat{u}(-h) \\
\hat{F}_{2}=-A \hat{\sigma}(h) & \hat{v}_{2}=-i \omega \hat{u}(h)
\end{array}
$$

This yields to Equation (6) and Equation (7) after some trigonometric manipulations, confirming the correctness of the circuit used. However, as such, the circuit is still incomplete: the quartz being piezoelectric, a third port electrical in nature should be added. Across a transformer, an electrical source connected to this port will be able to generate an acoustic wave and to be influenced by the mechanical properties of the device.

It can be shown that this three-port network satisfies the constitutive relations 
of piezoelectricity ; by introducing the piezoelectric stress coefficient $\tilde{e_{26}}$ and the dielectric constant at constant strain $\tilde{\varepsilon}$ of the quartz, these link the electric displacement $\hat{D}$ and the electric field $\hat{E}$ to the mechanical characteristics of the wave:

$$
\begin{aligned}
& \hat{\sigma}=\tilde{G} \frac{\mathrm{d} \hat{u}}{\mathrm{~d} z}-\frac{e_{26}}{\tilde{\varepsilon} \varepsilon_{0}} \hat{D} \\
& \hat{E}=-\frac{\tilde{e_{26}}}{\tilde{\varepsilon} \varepsilon_{0}} \frac{\mathrm{d} \hat{u}}{\mathrm{~d} z}-\frac{1}{\tilde{\varepsilon} \varepsilon_{0}} \hat{D}
\end{aligned}
$$

Finally, in practice and on one side, the QCM is in contact with the air, which has an acoustic wave impedance negligible compared with the one of the quartz. Therefore $\hat{F}_{1}=0$, and the equivalent circuit is short-circuited on the left. On the other side, the QCM is in contact with the material to characterise, with an acoustic impedance at the interface $\tilde{Z}_{L}$. Therefore $\hat{F}_{2} / \hat{v}_{2}=A \tilde{Z}_{L}$, and the equivalent circuit is closed with a resistance having this value. It remains in the circuit only the electrical port, which corresponds to the fact that the QCM can only be interrogated electrically through its electrodes, and not by any acoustic or mechanical mean.

\subsection{Resonance condition}

We can now use the Mason circuit to calculate the equivalent impedance of the circuit after the transformer, the motional impedance $\tilde{Z}_{\text {mot }}$. Although it does not have a clear physical significance, it is remarkable because it vanishes, by definition, if a resonance occurred; and in practice, the resonant frequency can be measured with a great precision. Using the Kirchhoff rules, the subscript $q$ indicating properties of the quartz, we have:

$$
\tilde{Z}_{m o t}=-\frac{\phi^{2}}{i \omega C_{0}}-\frac{i \tilde{Z}_{q}}{\sin \left(2 \tilde{k}_{q} h_{q}\right)}+\left(\left(i \tilde{Z}_{q} \tan \left(\tilde{k}_{q} h_{q}\right)\right)^{-1}+\left(i \tilde{Z}_{q} \tan \left(\tilde{k}_{q} h_{q}\right)+\tilde{Z}_{L}\right)^{-1}\right)^{-1}
$$

This relation can be simplified if we assume that the wavenumber $\tilde{k}_{q}$ is close to the ideal open-circuit wavenumber $\tilde{k}_{q, O C}=n \pi / 2 h_{q}$, solution of the equation $\tilde{Z}_{\text {mot }}=0$ when the QCM is fully immerged in the air without influence of the piezoelectric 
effect. Using a Taylor expansion of the previous relation yields:

$$
\tilde{Z}_{m o t} \approx \frac{1}{4}\left(-\frac{4 \phi^{2}}{i \omega C_{0}}+i n \pi \tilde{Z}_{q} \frac{\tilde{\omega}-\tilde{\omega}_{O C}}{\tilde{\omega}_{O C}}+\tilde{Z}_{L}\right)
$$

Applying this relation once in a reference state (the resonant angular frequency being called $\tilde{\omega}_{\text {sample}}$ ), with $\tilde{Z}_{L}=0$, and once in charge, the frequency shift obeys the following equation:

$$
\frac{\Delta \tilde{f}}{f_{0}}=\frac{\tilde{\omega}_{\text {ref }}-\tilde{\omega}_{\text {sample }}}{\frac{\tilde{\omega}_{O C}}{n}}=\frac{i}{\pi \tilde{Z}_{q}} \tilde{Z}_{L}
$$

This is a direct relation between frequencies measurement and the load impedance of the tested sample. To go further, some assumptions on the sample are required. If it is assumed, for instance, that it is semi-infinite, that is to say there is no right-traveling wave $\left(\hat{u}^{-}=0\right)$, Equations (4) and (5) yield:

$$
\frac{\hat{\sigma}(z)}{\hat{v}(z)}=\frac{i \omega \tilde{Z}_{\text {sample }} \hat{u}^{+} \exp \left(+i \hat{k}_{\text {sample }} z\right)}{i \omega \hat{u}^{+} \exp \left(+i \hat{k}_{\text {sample }} z\right)}=\tilde{Z}_{\text {sample }}
$$

Hence:

$$
\tilde{Z}_{L}=\frac{\hat{\sigma}\left(h_{q}\right)}{\hat{v}\left(h_{q}\right)}=\tilde{Z}_{\text {sample }}=\sqrt{\rho_{\text {sample }} \tilde{G}_{\text {sample }}}
$$

Given that the density of the sample is already known, the shear modulus can be deduced. Of course, the quantities characterizing the quartz in the previous relation $\left(\tilde{Z}_{q}\right.$ and $\left.f_{0}\right)$ are not known a priori. However, they can be evaluated by making an additional measurement with a sample in a well-known material, for instance water.

\subsection{Extraction of the resonance properties}

The impedance analyzer used in these experiments measures the electrical admittance $\tilde{Y}_{e l, m e a s u r e}$ as a function of the frequency. This paragraph explains how the complex resonance frequency can be deduced from this measurement. It can be 
shown that the linearization around the open-circuit frequency used previously allows getting a simpler representation of the equivalent circuit, using only standard electrical elements (resistance, inductance and conductance), which corresponds to the Butterworth-Van-Dyke model (Butterworth, 1914; Van Dyke, 1928), shown in Figure 2(d).

The Kirchhoff rules yield the electrical admittance of this circuit:

$$
\tilde{Y}_{\text {el,measure }}=i \omega C_{0}+\left(i \omega \bar{L}_{1}+\frac{1}{i \omega \bar{C}_{1}}+\bar{R}_{1}\right)^{-1}
$$

It is possible to fit the measurement $\tilde{Y}_{\text {el,measure }}(\omega)$ with this theoretical $\tilde{Y}_{e l}(\omega)$ and to deduce the complex resonance frequency from the values of the four elements, but it is helpful to use directly the following expanded Lorentzian functions which reduce the scatter in the fit parameters:

$$
\begin{aligned}
& \Re\left(\tilde{Y}_{e l}\right)=G_{e l, \max } \frac{f}{f_{r}}\left(\frac{\Gamma}{\left(f_{r}-f\right)^{2}+\Gamma^{2}} \cos (\theta)+\frac{f_{r}-f}{\left(f_{r}-f\right)^{2}+\Gamma^{2}} \sin (\theta)\right)+G_{e l, o f f} \\
& \Im\left(\tilde{Y}_{e l}\right)=B_{e l, \max } \frac{f}{f_{r}}\left(\frac{\Gamma}{\left(f_{r}-f\right)^{2}+\Gamma^{2}} \cos (\theta)-\frac{f_{r}-f}{\left(f_{r}-f\right)^{2}+\Gamma^{2}} \sin (\theta)\right)+B_{e l, o f f}
\end{aligned}
$$

Here, the fit parameters are the resonance frequency $f_{r}$, the half-bandwidth at half-height $\Gamma$, the maximal conductance $G_{e l, \max }$ and susceptance $B_{e l, \max }$, the conductance $G_{e l, o f f}$ and susceptance $B_{e l, o f f}$ offset, and the tilt angle $\theta$. The complex resonance frequency is then simply deduced from the first two parameters:

$$
\tilde{f}_{r}=f_{r}+i \Gamma
$$




\section{Application to the characterization of viscoelasticity of a polydimethyl- siloxane}

\subsection{Materials and methods}

The quartz crystal and its holder used in the experiments are the commercially available QCM200 (Stanford Research Systems, CA, USA). The crystal has a resonance frequency near $5 \mathrm{MHz}$ and a diameter of $2.54 \mathrm{~cm}$. It is covered with circular electrodes of titanium and gold. It is physically maintained with one Oring on both side and connected with the electrical interface via BNC connectors, which are then adapted to an SMA connection. The portable network analyzer is the miniVNA PRO (mini Radio Solutions, Germany), shown in Figure 3. It can be used in a wide range of frequencies, between $100 \mathrm{kHz}$ and $200 \mathrm{MHz}$ in step of $1 \mathrm{~Hz}$ and measure impedance between $1 \Omega$ and $1 \mathrm{k} \Omega$.

It is connected to a personal computer with a USB connection and controlled by a custom program, measuring continuously the admittance of the TSM resonator in frequencies centered on the resonance frequency $f_{r}$ with a span of $6 \cdot \Gamma$, the resonance half-bandwidth, both parameters being evaluated by a fit using the trust-region reflective method with a sufficiently small termination tolerance to ensure a coefficient of variation smaller than 3\% (Byrd et al., 2000). The whole setup is presented in Figure 4.

To minimize the variation of external parameters, the QCM is placed inside a thermostatic chamber at a given controlled temperature. The thermostatic chamber is located in a clean room which relative humidity is kept constant at approximately $50 \%$. The network analyzer is initially calibrated by the short-open-load method. The quartz crystal is washed with acetone, rinsed with water and dried with nitrogen before being placed inside its holder. Once the resonance frequency is stabilized, the crystal is loaded with a volume of $900 \mu \mathrm{L}$ of distilled water as the reference material, creating a layer thick enough to be considered as a semiinfinite medium. Finally, after stabilization of the resonance frequency, the water is removed and replaced by the same volume of $900 \mu \mathrm{L}$ of PDMS rubber RTV615 (Momentive Performance Materials Inc., NY, USA) in the liquid state, i.e. non cross-linked. Following the manufacturer's recommendations, it was obtained by weighting out ten parts of the uncured rubber with one part of a curing agent. The components were then thoroughly mixed together, and degassed with exposition to partial vacuum for about ten minutes, in order to remove the entrapped air and guarantee an homogenous cured product. 
Thus, the evolution of the viscoelastic properties was measured continuously from the liquid (uncross-linked) state to the solid (cross-linked) state. For information, Table 1 provides some mechanical properties obtained once the material is cured.

Table 1

Mechanical properties of the RTV615 silicone rubber cured $1 \mathrm{hr}$ at $100{ }^{\circ} \mathrm{C}$

\begin{tabular}{|c|c|}
\hline Property & cured RTV615 \\
\hline Hardness [Shore A] & 44 \\
Tensile Strength $\left[\mathrm{kg} / \mathrm{cm}^{2}\right]$ & 65 \\
Elongation at break [\%] & 120 \\
Shrinkage [\%] & 0.2 \\
\hline
\end{tabular}

The temperature of the PDMS sample surface was measured by using a CT-SF15C3 Micro-Epsilon pyrometer, 15:1 in optical resolution, 8-14 $\mu \mathrm{m}$ in spectral range, $150 \mathrm{~ms}$ in response time and $0.1 \mathrm{~K}$ in thermal resolution.

\subsection{Results and discussion}

Figure 5 gives the evolution of the viscoelastic properties in terms of shear modulus G' (Fig. 5(a)) and loss factor $\tan (\delta)$ (Fig. 5(b)) versus time. Results obtained at $25^{\circ} \mathrm{C}, 50^{\circ} \mathrm{C}$ and $80^{\circ} \mathrm{C}$ appears in red, blue and orange colors, respectively. First, the analysis focuses on evolution of the viscoelastic properties at the lowest temperature. At $25^{\circ} \mathrm{C}$, the shear modulus evolution is strongly nonlinear from $4 \mathrm{MPa}$ for the liquid state (only slightly cross-linked) to the stabilized value of $12 \mathrm{MPa}$ for the solid state. Several regimes can be distinguished to describe the shear modulus evolution, as illustrated by the dashed line in Fig. 5(a). During the first ten hours, G' slowly increases, then strongly increases (from 10 to $17 \mathrm{hr}$ ), slowly increases (from 17 to $27 \mathrm{hr}$ ), before being almost stabilized at the $35^{\text {th }} \mathrm{hr}$. The loss factor decreases and evolves in a same nonlinear way from 1.6 to 0.8 (see Fig. 5(b)). The results show that in the megahertz domain the elastic modulus and the loss factor are high compared to values obtained with classical dynamic mechanical analysis (DMA), see for instance the work by Rey et al. (2013) on PDMS under tension loading condition. This is expected as the frequency is increased. This measurement at $25^{\circ} \mathrm{C}$ is consistent with the data provided by the material supplier, who states that the material is sufficiently cured in 24 hours to permit handling.

This evolution in the viscoelastic properties is directly linked with the cross-linking kinetics. In lower frequency domains, modeling approaches have been developed 
to predict the shear equilibrium modulus, most of them being based on the phenomenological models by Langley (1959) and Dossin and Graessley (1979). In these models, the contribution of chemical crosslinks is distinguished from that of trapped physical entanglements. At much higher frequencies, leading to very low displacement amplitudes, the topological contribution to the shear modulus seems difficult to evaluate and the subsequent prediction of the shear equilibrium modulus is therefore delicate. Further investigations are therefore required to model the effects of the kinetics of cross-linking on the viscoelastic properties in the megahertz domain.

When the temperature is increased, the curves obtained at $50{ }^{\circ} \mathrm{C}$ and $80^{\circ} \mathrm{C}$ highlight that the higher the temperature, the faster the evolution of viscoelastic properties and their stabilization. This result is expected since increasing temperature accelerates cross-linking. Furthermore, the first regime described at the lowest temperature is not observed anymore at the highest temperature at $80{ }^{\circ} \mathrm{C}$ and is reduced to the first hour at $50^{\circ} \mathrm{C}$.

In Figure 6, the time at viscoelastic properties stabilization for the three crosslinking temperatures have been compared to the times provided by the supplier (see the data-sheet curve in blue). The times measured and provided are very close.

Finally, temperature measurements were carried out at the material disc surface during the test. The results obtained at 25,50 and $80^{\circ} \mathrm{C}$ are presented in Figure 7 in blue color, in red color and in orange color, respectively. Before analyzing the sample temperature evolution, it is necessary to recall that the QCM is placed inside the thermostatic chamber at the desired controlled temperature. The thermostatic chamber door has to be opened to place the quartz crystal inside its holder. After stabilization of the resonance frequency, the water used to calibrate the measurement is removed and replaced by the same volume of $900 \mu \mathrm{L}$ of PDMS rubber RTV615 (the door is re-opened). Therefore, once the door is closed, a certain time is needed to achieve the thermal stabilization. At $25^{\circ} \mathrm{C}$, as the temperature is close to the ambient temperature of the laboratory, the temperature of the sample (see the blue curve in Fig. 7) is quite constant. At the highest temperatures, the door opening induces a significant effect: 40 minutes are needed for stabilizing the sample's temperature.

In a first approximation, the temperature change due to heat diffusion should follow a Fourier law, meaning that it should evolve as an exponential function of time. Nevertheless, this is not the case at $50{ }^{\circ} \mathrm{C}$ and even more at $80^{\circ} \mathrm{C}$. This is the signature of cross-linking kinetics, cross-linking being exothermal. The heat produced by cross-linking increases, becomes detectable by the pyrometer and the 
temperature curve shape changes.

At this stage, an important question arises. Indeed, the measurements have shown that temperature influences the cross-linking kinetics. Due to viscosity, the higher the material temperature, the lower (higher) the elastic (viscous) modulus (see Figs 5(a) and 5(b)). But are the final viscoelastic properties the same? In other words, does the change in the kinetics affect the final properties? To answer this question, the viscoelastic properties of samples cross-linked at 50 and $80^{\circ} \mathrm{C}$ have been measured at $25^{\circ} \mathrm{C}$. Table 2 summarizes results obtained. Results show that

Table 2

Viscoelastic properties measured at $25^{\circ} \mathrm{C}$

\begin{tabular}{|c|c|c|}
\hline Cross-linking temperature $\left[{ }^{\circ} \mathrm{C}\right]$ & $\mathrm{G}^{\prime}[\mathrm{MPa}]$ & $\tan \delta[-]$ \\
\hline 25 & 12 & 0.8 \\
50 & 12.6 & 0.69 \\
80 & 13.9 & 0.65 \\
\hline
\end{tabular}

the higher the cross-linking temperature, the higher the shear modulus and the lower the loss factor, meaning that the cross-linking kinetics has clearly an effect on the final viscoelastic properties.

\section{Conclusion}

The present paper aimed at presenting the QCM as a relevant tool for measuring the viscoelastic properties of rubbers in the megahertz domain. The advantage of such a technique is that the measurement can be carried out for liquids and for solids as well, i.e. from the uncross-linked to the cross-linked state. The volume of the material needed is small compared to classical characterizations. This technique is well adapted to measurements in an oven, i.e. at different temperatures. It has been used to successfully characterized the viscoelastic properties of a polydimethylsiloxane and their evolution during cross-linking at three different temperatures : 25, 50 and $80{ }^{\circ} \mathrm{C}$. Results have shown that the final viscoelastic properties depend on the temperature during cross-linking. The higher the crosslinking temperature, the higher (lower) the final elastic (viscous) modulus at 25 ${ }^{\circ} \mathrm{C}$. Therefore, increasing the temperature accelerates the cross-linking kinetics, but also influences the final viscoelastic properties (the material becomes stiffer and the loss factor decreases). 


\section{References}

Arnau, A. (2008). A review of interface electronic systems for AT-cut quartz crystal microbalance applications in liquids. Sensors, 8, 370-411.

Becker, B. and Cooper, M. A. (2011). A survey of the 2006-2009 quartz crystal microbalance biosensor literature. Journal of Molecular Recognition, 24, 754787.

Butterworth, S. (1914). On electrically-maintained vibrations. Proceedings of the Physical Society of London, 27, 410.

Byrd, R. H., Gilbert, J. C., and Nocedal, J. (2000). A trust region method based on interior point techniques for nonlinear programming. Mathematical Programming, 89, 149-185.

Cheeke, J. and Wang, Z. (1999). Acoustic wave gas sensors. Sensors and Actuators $B, \mathbf{5 9}, 146-153$.

Dossin, L. M. and Graessley (1979). Rubber elasticity of well-characterized polybutadiene networks. Macromolecules, 12, 123-130.

Hinsberg, W., Willson, C., and Kanazawa, K. K. (1986). Measurement of ThinFilm Dissolution Kinetics Using a Quartz Crystal Microbalance. Journal of The Electrochemical Society, 133, 1448-1451.

Holt, R. C., Gouws, G. J., and Zhen, J. Z. (2006). Measurement of polymer shear modulus using thickness shear acoustic waves. Current Applied Physics, 6, 334339.

Johannsmann, D. (2015). The Quartz Crystal Microbalance in Soft Matter Research: Fundamentals and Modeling. Soft and Biological Matter. Springer International Publishing, 1 edition.

Kanazawa, K. K. and Gordon, J. G. (1985). The oscillation frequency of a quartz resonator in contact with liquid. Analytica Chimica Acta, 175, 99-105.

Langley, N. R. (1959). Elastically effective strand density in polymer networks. Macromolecules, 1, 348-352.

Lucklum, R., Behling, C., and Hauptmann, P. (2000). Gravimetric and nongravimetric chemical quartz crystal resonators. Sensors and Actuators B, 65, $227-283$.

Mason, W. P. and Baerwald, H. (1951). Piezoelectric crystals and their applications to ultrasonics. Physics Today, 4, 23.

Rey, T., Chagnon, G., Le Cam, J.-B., and Favier, D. (2013). Influence of the temperature on the mechanical behaviour of filled and unfilled silicone rubbers. Polymer Testing, 32, 492-501.

Sauerbrey, G. (1959). Verwendung von schwingquarzen zur wägung dünner schichten und zur mikrowägung. Zeitschrift fur Physik, 155, 206-222.

Serfaty, S., Griesmar, P., Le Huu, J., and Caplain, E. (2012). Piezoelectric sensor for the detection and characterization of at least one biochemical element. 
Si, P., Mortensen, J., Komolov, A., Denborg, J., and Møller, P. J. (2007). Polymer coated quartz crystal microbalance sensors for detection of volatile organic compounds in gas mixtures. Analytica chimica acta, 597, 223-230.

Stockbridge, C. (1966a). Effects of gas pressure on quartz crystal microbalances. Vacuum Microbalance Techniques, 5, 147-178.

Stockbridge, C. (1966b). Resonance frequency versus mass added to quartz crystals. Vacuum Microbalance Techniques, 5, 193.

Van Dyke, K. (1928). The piezo-electric resonator and its equivalent network. Proceedings of the Institute of Radio Engineers, 16, 742-764.

Wang, H., Zhang, Y., Yan, B., Liu, L., Wang, S., Shen, G., and Yu, R. (2006). Rapid, simple, and sensitive immunoagglutination assay with $\mathrm{SiO} 2$ particles and quartz crystal microbalance for quantifying Schistosoma japonicum antibodies. Clinical chemistry, 52, 2065-2071.

Yu, J.-S., Liao, H.-X., Gerdon, A. E., Huffman, B., Scearce, R. M., McAdams, M., Alam, S. M., Popernack, P. M., Sullivan, N. J., Wright, D., and others (2006). Detection of Ebola virus envelope using monoclonal and polyclonal antibodies in ELISA, surface plasmon resonance and a quartz crystal microbalance immunosensor. Journal of virological methods, 137, 219-228. 

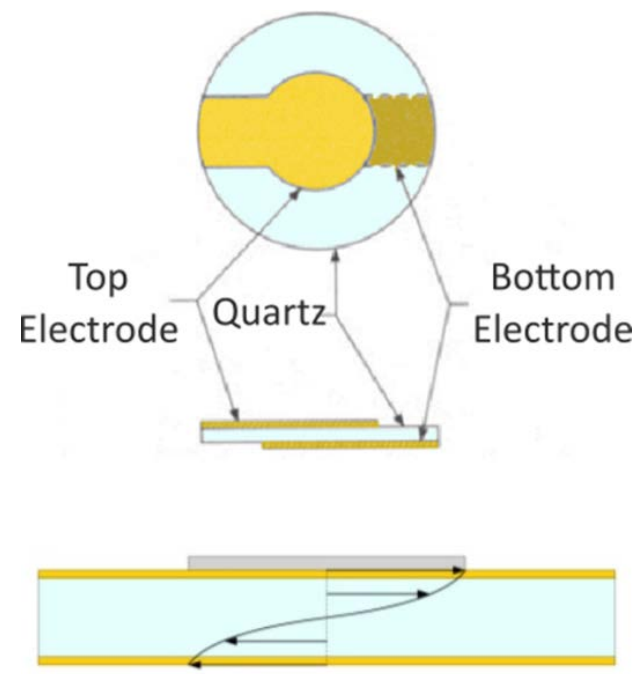

Figure 1. Two-port network representation of a layer in which propagate a shear-wave. 


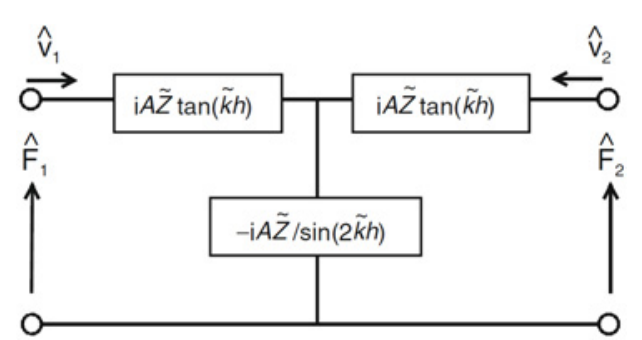

(a) Two-port network representation of a layer in which propagate a shear-wave.

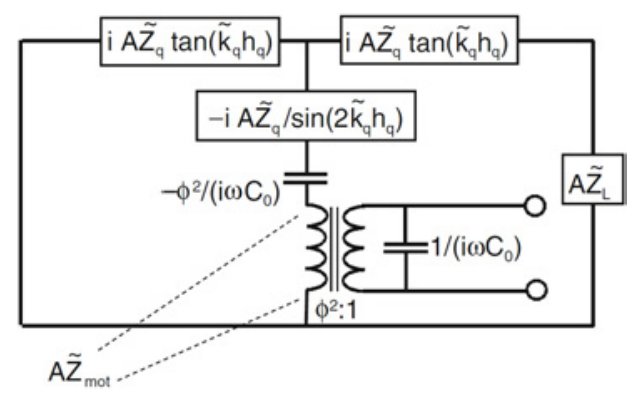

(c) One-port network representation of a QCM in contact with the air on one side and a specific sample on the other.

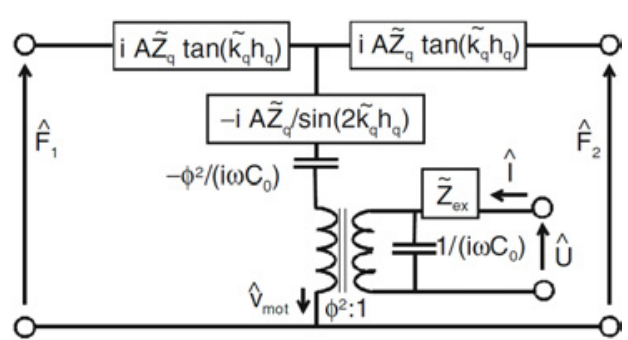

(b) Three-port network representation of a piezoelectric layer in which propagate a shear-wave.

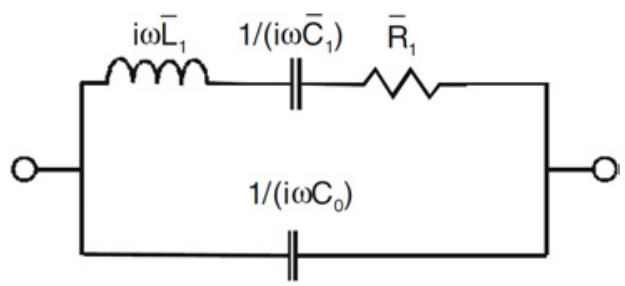

(d) Electrical circuit associated with the Butterworth-Van-Dyke model.

Figure 2. Schematic diagrams of networks and circuits 


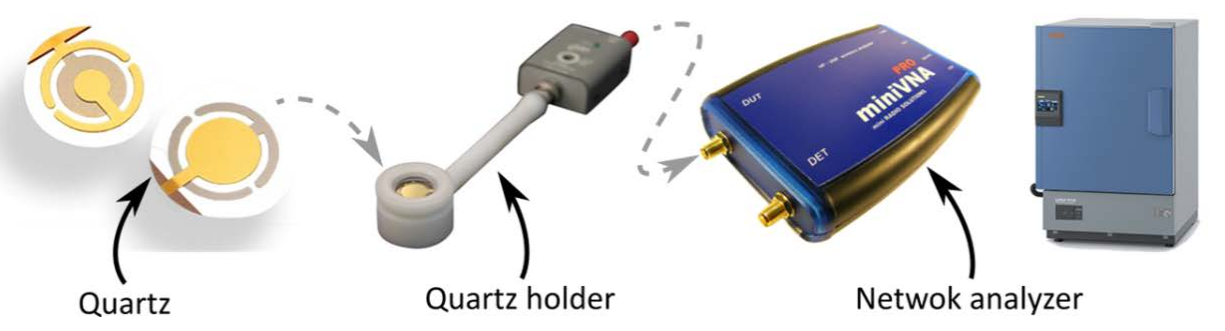

Figure 3. Photographs of the various components of the experimental setup. 


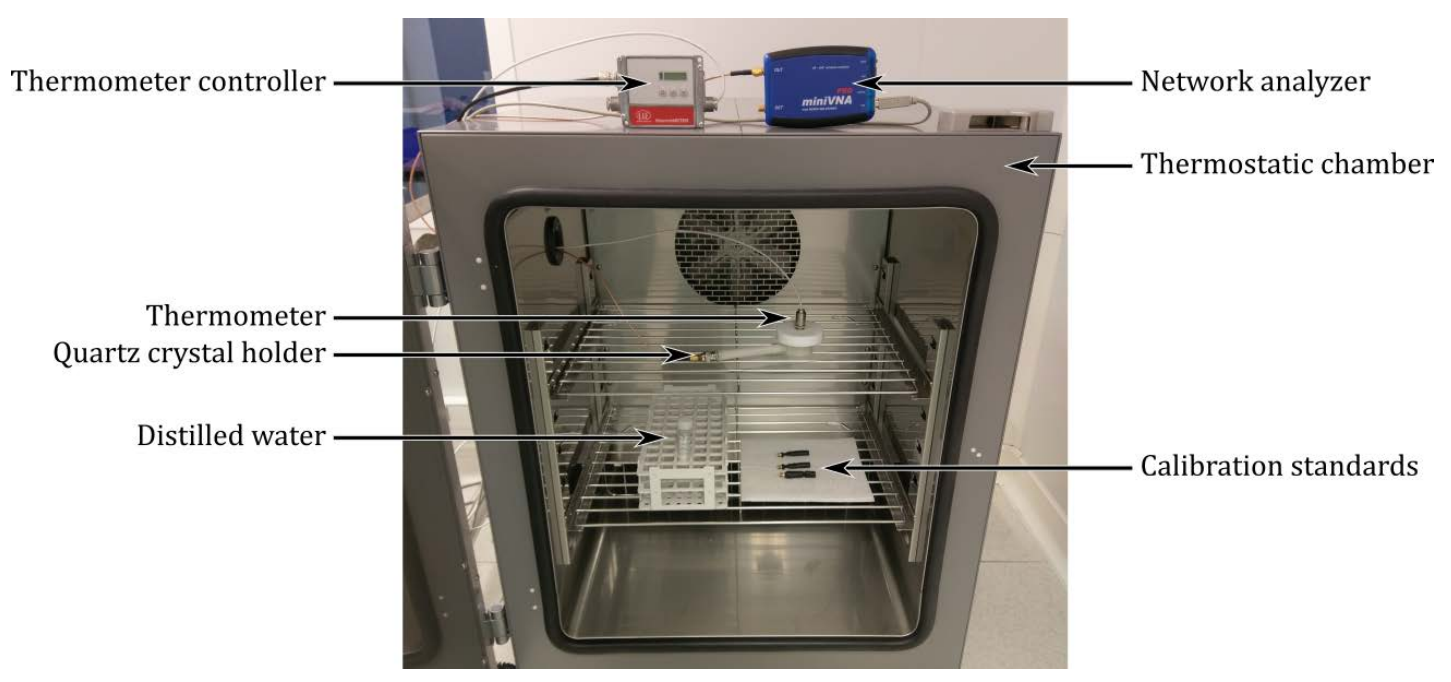

(a) Global view of the experimental setup.

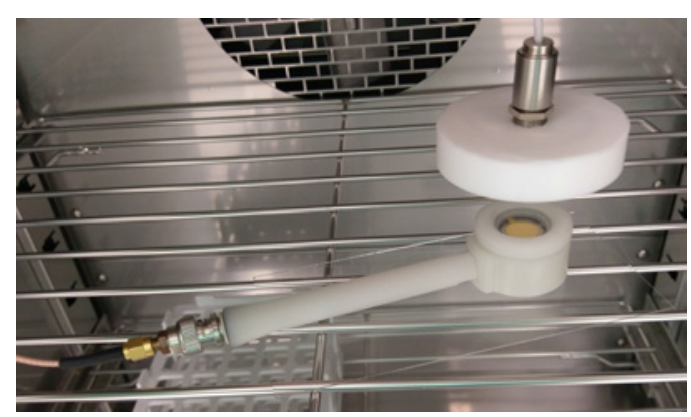

(b) Close-up view of the experimental setup.

Figure 4. Photographs of the experimental setup. 


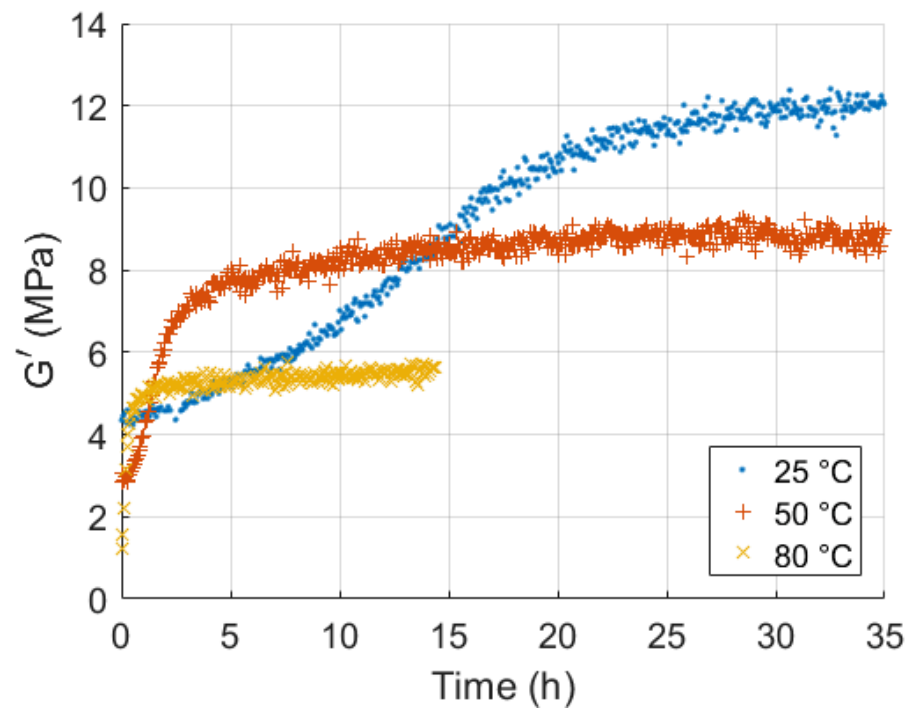

(a) shear modulus $G$ '.

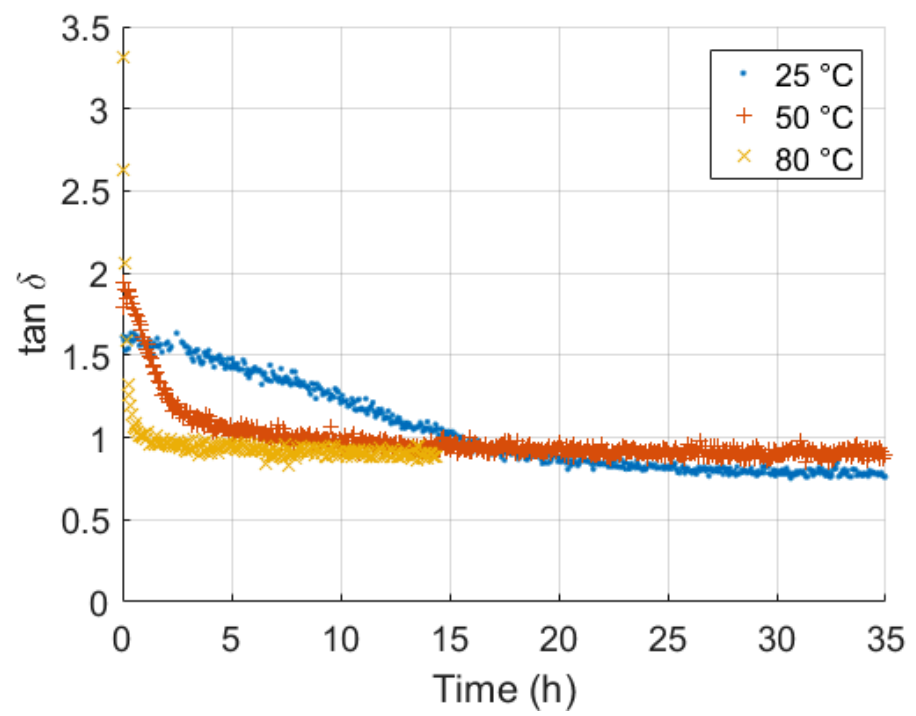

(b) loss factor $\tan (\delta)$.

Figure 5. Evolution of the viscoelastic properties at different ambient temperatures, respectively $25^{\circ} \mathrm{C}$ (in blue color), $50^{\circ} \mathrm{C}$ (in red color) and $80^{\circ} \mathrm{C}$ (in orange color). 


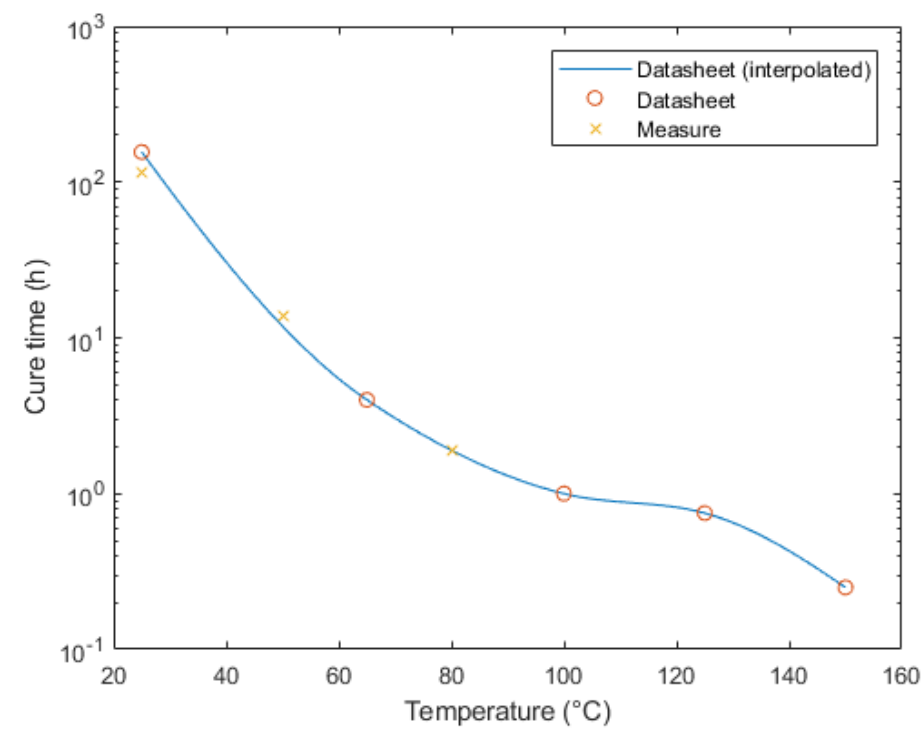

Figure 6. Time at viscoelastic properties stabilization measured and provided by the supplier. 


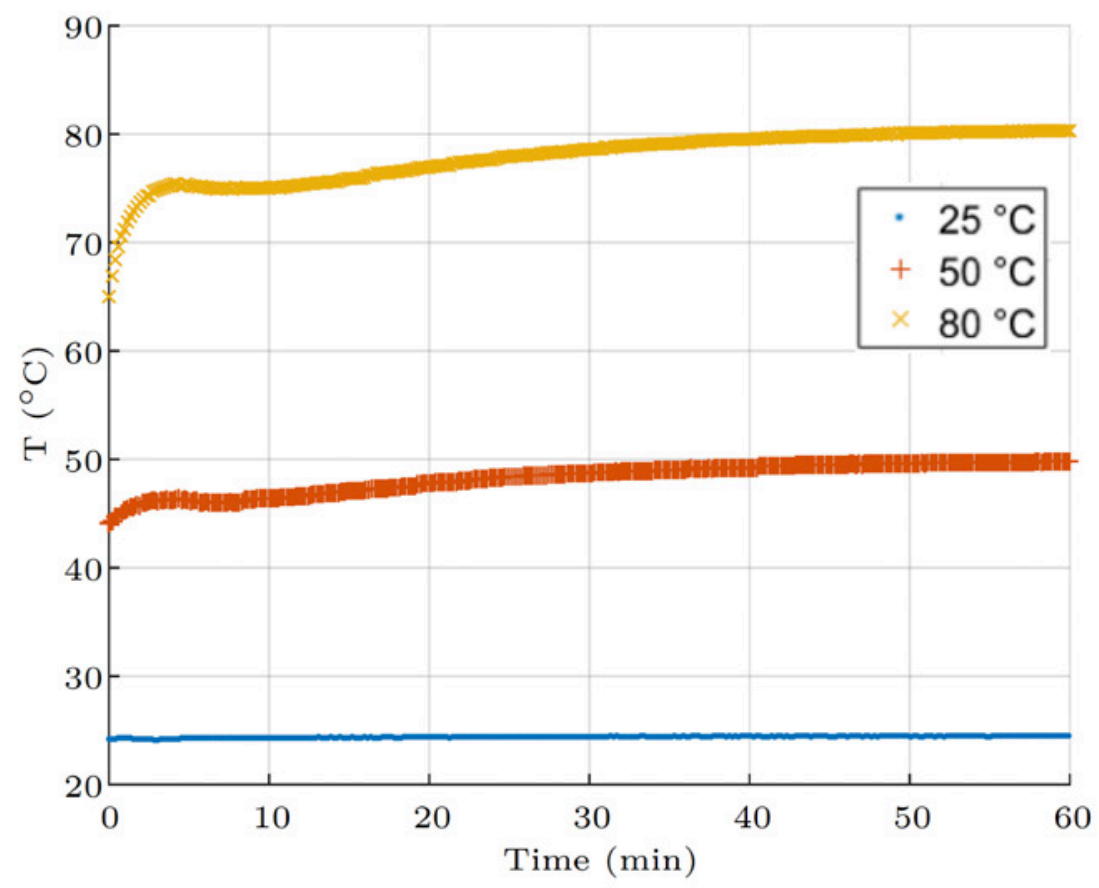

Figure 7. Evolution of the measured temperature of the sample during the experiment at different ambient temperatures, respectively $25^{\circ} \mathrm{C}$ (in blue color), $50^{\circ} \mathrm{C}$ (in red color) and $80^{\circ} \mathrm{C}$ (in orange color). The temperature does not vary after one hour. 